\title{
Co-infection of other respiratory pathogens in COVID-19: associated factors analysis and prognostic impact assessment
}

\author{
Xiaowen $\mathrm{Hu}^{1}$, Feng zhang ${ }^{1}$, Liyan Dong ${ }^{1}$, Bei $\mathrm{Pan}^{1}$, Wei Ni${ }^{2}$, Ruqin $\mathrm{Gao}^{1}$, Zhaoguo Wang ${ }^{1}$, \\ and Fachun Jiang ${ }^{1}$ \\ ${ }^{1}$ Qingdao Municipal Center for Disease Control and Prevention \\ ${ }^{2}$ Qingdao Women and Children's Hospital
}

August 28, 2020

\begin{abstract}
The possibility of co-infection with other respiratory pathogens in COVID-19 remains unclear. This study aims to expand the knowledge of distribution and associated factors of co-infection in COVID-19 patients, and to assess the impact of co-infection on COVID-19 prognosis. Both univariate and multivariate analysis were performed to identify independent factors for co-infection. Cox regression was conducted to detect the association between co-infection and negative conversion after controlling other related factors. The rate of co-infection with at least one of other respiratory pathogens was $76.4 \% .83 .3 \%$ of co-infection cases were detected bacterial co-infection, followed by $31.0 \%$ with viral co-infection. Over $70 \%$ of neutrophils proportion (OR: 4.563 ; 95\% CI: 1.116-18.648) was independently factors for bacterial co-infection, but fever (HR: 4.506; 95\%CI: 1.044-19.441) and chest tightness (OR: 0.106; 95\%CI: 0.015-0.743) for viral co-infection. The strongest promotion of negative conversion was detected with co-infection of only viruses (HR: 4.039; 95\%CI: 1.238-13.177), and the weakest was found for co-infection of only bacteria (HR: 2.909; 95\%CI: 1.308-6.472). The promotion of mixed bacteria and viruses was between co-infection of only bacteria and only viruses (OR: 3.242; 95\%CI: 1.171-8.977). A higher proportion of bacterial co-infection is detected in comparison with viral co-infection among COVID-19 patients. Over $70 \%$ of neutrophils proportion, fever and chest tightness are independent factors associated with specific co-infection in SARS-CoV-2 infection. Additionally, different types of co-infection could variously result in a promoted negative conversion of COVID-19.
\end{abstract}

\section{Full Title}

Co-infection of other respiratory pathogens in COVID-19: associated factors analysis and prognostic impact assessment

\section{Running title}

Co-infection in COVID-19 patients

\section{Authors}

Xiaowen $\mathrm{Hu}^{1 *}$, Feng Zhang ${ }^{1 *}$, Liyan Dong ${ }^{1}$, Bei $\operatorname{Pan}^{1}$, Wei Ni²\#, Ruqin Gao ${ }^{1 \#}$, Zhaoguo Wang ${ }^{1 \#}$, Fachun Jiang ${ }^{1 \#}$

\section{Affiliations}

${ }^{1}$ Qingdao Municipal Center for Disease Control and Prevention, Qingdao Institute of Prevention Medicine, Qingdao, Shandong Province, China.

${ }^{2}$ Qingdao Women and Children's Hospital, Qingdao University, Qingdao, Shandong Province, China

\section{*Contributed equally}




\section{\#Correspondence}

Fachun Jiang, Dr: Qingdao Municipal Center for Disease Control and Prevention, Qingdao Institute of Prevention Medicine, Qingdao City, Shandong Province, P.R.China. No.175 Shandong Road, 266033, China. E-mail: 13583206500@163.com

Zhaoguo Wang, Dr: Qingdao Municipal Center for Disease Control and Prevention, Qingdao Institute of Prevention Medicine, Qingdao City, Shandong Province, P.R.China. No.175 Shandong Road, 266033, China. E-mail: 470421859@qq.com

Ruqin Gao, MM: Qingdao Municipal Center for Disease Control and Prevention, Qingdao Institute of Prevention Medicine, Qingdao City, Shandong Province, P.R.China. No.175 Shandong Road, 266033, China. E-mail: ruqingao123@sina.com

Wei Ni, MM: Qingdao Women and Children's Hospital, Qingdao University, Qingdao, Shandong Province, P.R.China, No.6 Tongfu Road, 266000, Qingdao, China. E-mail: 115438779@qq.com

\section{Summary}

The possibility of co-infection with other respiratory pathogens in COVID-19 remains unclear. This study aims to expand the knowledge of distribution and associated factors of co-infection in COVID-19 patients, and to assess the impact of co-infection on COVID-19 prognosis. Both univariate and multivariate analysis were performed to identify independent factors for co-infection. Cox regression was conducted to detect the association between co-infection and negative conversion after controlling other related factors. The rate of co-infection with at least one of other respiratory pathogens was $76.4 \% .83 .3 \%$ of co-infection cases were detected bacterial co-infection, followed by $31.0 \%$ with viral co-infection. Over $70 \%$ of neutrophils proportion (OR: 4.563; 95\%CI: 1.116-18.648) was independently factors for bacterial co-infection, but fever (HR: 4.506; 95\%CI: 1.044-19.441) and chest tightness (OR: 0.106; 95\%CI: 0.015-0.743) for viral co-infection. The strongest promotion of negative conversion was detected with co-infection of only viruses (HR: 4.039; 95\%CI: 1.238-13.177), and the weakest was found for co-infection of only bacteria (HR: 2.909; 95\%CI: 1.308-6.472). The promotion of mixed bacteria and viruses was between co-infection of only bacteria and only viruses (OR: 3.242; 95\%CI: 1.171-8.977). A higher proportion of bacterial co-infection is detected in comparison with viral co-infection among COVID-19 patients. Over 70\% of neutrophils proportion, fever and chest tightness are independent factors associated with specific co-infection in SARS-CoV-2 infection. Additionally, different types of co-infection could variously result in a promoted negative conversion of COVID-19.

Keywords: Co-infection; COVID-19; Respiratory pathogens; independent factors; Negative conversion

\section{Introduction}

Since a cluster of coronavirus disease 2019 (COVID-19) cases were first reported in Wuhan, China, in December 2019, it has become a global threat and spread rapidly across more than 200 countries and regions (WHO, 2020). A striking aspect of COVID-19 is that the disease became a pandemic in several months, resulting in over 16 million cases and 600 thousand deaths worldwide as of 30 July (WHO, 2020). Although we learned more about COVID-19 and severe acute respiratory syndrome coronavirus 2 (SARS-CoV-2) in the past several months depending on the modern advanced technology, some issues, such as a useful strategy to prevent disease spread, transmission route, and effective treatments, have been still severe challenges until now. Additionally, the possibility of co-infection with other respiratory pathogens remains unclear. This should be an important concern for clinicians in the management of COVID-19.

Generally, co-infection has been already detected during the epidemic of previous emerging infectious diseases, including severe acute respiratory syndrome (SARS) and Middle East respiratory syndrome (MERS). Previous serological evidence revealed that the incidences of acute or recent Chlamydophila pneumonia (CP) or Mycoplasma pneumonia (MP) infection in SARS patients were $30 \%$ and $9 \%$, respectively (Zahariadis et al., 2006). In Hong Kong, a major nosocomial outbreak of SARS revealed co-infection of human metapneu- 
movirus (MPV) (Lee et al., 2007). Moreover, co-infection of Middle East respiratory syndrome coronavirus (MERS-CoV) with influenza has been reported, and it demonstrated that $18 \%$ critically ill patients with MERS-CoV had bacterial co-infection, followed by $5 \%$ with viral co-infection, in a multicenter retrospective cohort study (Alfaraj et al., 2017; Arabi et al., 2017). However, there is limited data regarding co-infection of other respiratory pathogens in COVID-19 patients, as well as the impact of co-infection on COVID-19 prognosis.

It is considered that the recognition of SARS-CoV-2 infection is important as it enables the implementation of appropriate infection control measures and possible promising antiviral therapy, but clinicians should not neglect the possibility of SARS-CoV-2 co-infection (Lai et al., 2020). Therefore, we aim to expand the knowledge of distribution and associated factors of co-infection in COVID-19 patients, and assess the impact of co-infection on COVID-19 prognosis.

\section{Methods}

\subsection{Participants}

We recruited patients with laboratory-confirmed COVID-19 who admitted to the designated hospitals in Qingdao from January 22 to February 14, 2020. The detection of SARS-CoV-2 and other respiratory pathogens for all patients were tested at the onset of COVID-19. Data were collected onto standardized forms through interviews of infected persons. Epidemiological, demographic, clinical, laboratory, treatment and prognosis data were extracted from the interview as well as field reports. All patients were diagnosed with COVID-19 according to New Coronavirus Pneumonia Diagnosis and Treatment Plan (NHCPRC, 2020a; NHCPRC, 2020b; NHCPRC, 2020c).

This study was approved by the Ethics Commission of Qingdao Municipal Center for Disease Control and Prevention (Qingdao CDC) and written informed consent was waived considering the emergency of infectious disease.

\subsection{Detection of SARS-CoV-2 in respiratory samples}

The doctors collected the samples by rubbing 2 of the patient's nostrils and the posterior oropharynx using separate cotton-tipped swabs. After sampling, the swabs were collected into a single virus collection tube which containing virus preservation solution and sent to the laboratory of Qingdao CDC via dedicated channel. Samples were stored at $-20^{\circ} \mathrm{C}$ if not be analyzed immediately. All samples after sampling were transferred to QMCDCP for SARS-CoV-2 detection. Tests were carried out in biosafety level 2 facilities, using a commercial Novel Coronavirus (2019-nCoV) Nucleic Acid Detection Kit (Shanghai BioGerm Medical Techonology Co., LTD) in a total reaction volume of $25 \mu \mathrm{L}$, targeting SARS-CoV-2 virus frame1ab (ORF). Viral ribonucleic acid (RNA) was extracted from sample material and collected in elution buffer, and then underwent real-time reverse-transcription-polymerase-chain-reaction (RT-PCR) with SARS-CoV-2-specific primers and probes. Detailed laboratory procedures were referenced and described elsewhere (Hu et al., 2020; Hu et al., 2020).

\subsection{Detection of other respiratory pathogens}

The common respiratory pathogens were detected with RT-PCR based on general procedure. 16 bacterial and 13 viral pathogens were detected in this study, including Mycoplasma pneumoniae (MP), Moraxella catarrhalis (MC), Chlamydophila pneumonia (CP),Streptococcus pneumonia (SP), Haemophilus influenzae (HI),Staphyloccocus aureus (SA), Pseudomonas aeruginosa (PA), Moraxella catarrhalis (MA), Klebsiella pneumoniae (KP), Legionella pneumophila (LP), Bordetella pertussis (BP), Mycobacterium avium (AV), Mycobacterium tuberculosis (TB), Acinetobacter baumannii (AB), Bordetella parapertussis(BPP), Stenotrophomonas maltophilia (SMP), Serratia marcescens (SM), influenza A (IVF-A), influenza B (IVF-B), human respiratory syncytial virus (RSV), parainfluenza (PIV, contended 1,2, 3 and 4 four types), human metapneumovirus (HMPV), coronaviruses 229E, OC43 and NL63 (HCoV-229E, HCoV-OC43 and HCoVNL63), human bocavirus adenovirus (HBoV), human adenovirus (HAdV), human rhinovirus (HRV), human enterovirus (HEV) and MERS-CoV, were also detected using pathogen specific one-step respiratory viruses 
multiplex RT-PCR detection Kit (Neuro-Hemin Biotech) and pneumobacter multiplex PCR detection Kit (Neuro-Hemin Biotech). The reliability of detection reagent was verified by a separate fluorescent real-time RT-PCR kit.

\subsection{Statistical analysis}

In our study, co-infection of other respiratory pathogens in COVID-19 patients, was the outcome measure and presented as the categorical variable. In order to determine the independent factors of co-infection, univariate and multivariate analysis were performed in sequence. Mann-Whitney $U$ test, $\chi^{2}$ test or Fisher's exact test was first conducted to compare differences between groups with and without co-infection of other respiratory pathogens in COVID-19 patients. Next, factors with statistical significance $(P<0.05)$ were further analyzed using the Logistic regression model, and their odds ratio (OR) were calculated. According to the types of respiratory pathogens, co-infection with bacteria and viruses were separately analyzed through the above statistical process.

Additionally, we used Cox regression to assess the impact of co-infection on prognosis of COVID-19. In our study, we focused on the negative conversion of SARS-CoV-2 to determine the variation of viral shedding duration associated with co-infection in COVID-19 patients. Co-infection was introduced into the Cox regression model, which was set as a categorical variable presented by no co-infection (endowed by 0 ), coinfection of only bacteria (endowed by 1), co-infection of only viruses (endowed by 2) and co-infection of mixed bacteria and viruses (endowed by 3), respectively. A previous study suggested that age older than 45 years and chest tightness are independent factors affecting negative conversion of SARS-CoV-2 RNA (Hu et al., 2020). Therefore, age older than 45 years and chest tightness were also introduced to control their impacts.

Continuous and categorical variables were presented as median (interquartile range, IQR) and n (\%), respectively. A P value less than 0.05 (two-tailed) was considered statistically significant. Analyses were performed using SPSS software (version 22.0)and R software (version 3.6.3).

\section{Results}

\subsection{Characteristics of COVID-19 patients}

A total of 55 patients hospitalized with COVID-19 were included in this study, with a median age of patients was 45 years (IQR: 32-58). Among confirmed cases, 31 were female, which accounted for $56.4 \%$, followed by 24 male cases for $43.6 \%$.

The median duration from disease onset to hospital admission was 2 (IQR: 1-5) days, with a median of 19 days from illness onset to hospital discharge (IQR: 16-25.5). As shown in Table 1, 80\% (44/55) of the COVID-19 patients were non-severe (mild to moderate cases), and $20 \%(11 / 55)$ were severe cases. The most common symptoms at onset of the disease were fever (76.4\%) and non-productive cough (38.2\%). 13 patients had underlying diseases, cardiovascular and cerebrovascular diseases, endocrine disease, gastrointestinal disease, respiratory disease and neurological disorder.

On admission, 5 patients had leucopenia (leucocyte count count $<4 \times 10^{9} / \mathrm{L}$ ). 15 patients had increases proportion of neutrophils $(>70 \%)$, and 7 patients showed elevated lymphocyte percentage $(>40 \%)$. Abnormalities in chest computed tomograms (CT) were detected in 44 patients (80\%). The most pattern of CT changes was high-density shadow (61.4\%).

All patients received standard care including supportive and antiviral treatment according to the latest clinical guidelines (NHCPRC, 2020a; NHCPRC, 2020b; NHCPRC, 2020c). Antibiotics were only prescribed to patients who were at risk of presented with bacterial infection. Patients with symptoms of respiratory distress received oxygen therapy. Corticosteroids were used shortly to inhibit inflammatory cascade in patients with progressing disease. Critically ill patients were transferred to intensive care unit. More detailed information was reported in Table 1 .

\subsection{Co-infection in COVID-19 patients}


Among all the 55 COVID-19 patients, 42 cases $(42 / 55,76.4 \%)$ had RT-PCR positive detection against at least one of other respiratory pathogens, including bacterial co-infection $(35 / 55,63.6 \%)$ and viral co-infection $(13 / 55,23.6 \%)$. As shown in Figure 1 , four types of bacteria and five types of viruses were detected in COVID-19 patients. Among co-infected cases, 83.3\% (35/42) were detected with bacterial pathogens, which was as follows: SP $(25 / 42,59.5 \%)$, HI $(16 / 42,38.1 \%)$, MC $(6 / 42,14.3 \%)$, and PA (4/42, 9.5\%). Moreover, $31.0 \%(13 / 42)$ of co-infected patients were detected with viral pathogens, which included IFV-B (8/42, $19.0 \%)$, HRV (3/42, 7.1\%), IFV-A (1/42, 2.4\%), RSV (1/42, 2.4\%) and PIV3 (1/42, $2.4 \%)$. In addition, $64.3 \%(27 / 42)$ of co-infected cases were detected with a single pathogen, followed by $35.7 \%(15 / 42)$ with more than one pathogen. The common combination of co-infection (more than one pathogen) were $\mathrm{SP}+\mathrm{HI}+\mathrm{MC}$ $(3 / 42,7.1 \%), \mathrm{SP}+\mathrm{HI}(2 / 42,4.8 \%), \mathrm{SP}+\mathrm{HI}+\mathrm{PA}(2 / 42,4.8 \%)$ and $\mathrm{SP}+\mathrm{MC}(2 / 42,4.8 \%)$. More information regarding the distribution of co-infection of other respiratory pathogens in COVID-19 patients was showed in Figure 1 .

\subsection{Independent factors of co-infection}

Table 1 summarized the results of univariate analysis. We evaluated the effect of each factor on co-infection of other respiratory pathogens among COVID-19 patients by Mann-Whitney U test, $\chi^{2}$ test, or Fisher's exact test. Results from multivariate Logistic regression model were shown in Table $\mathbf{2}$. To the bacterial coinfection, sex, chest tightness and neutrophil percentage were significantly related to co-infection of bacterial pathogens. Results from multivariate Logistic regression, introduced the three above factors, revealed that over $70 \%$ of neutrophils proportion (OR: $4.563 ; 95 \%$ CI: 1.116-18.648) was independently factors. Moreover, according to results of univariate analysis, fever and chest tightness were significantly related to co-infection of viral pathogens, and after introducing two of them into Logistic regression model, fever (OR: 4.506; 95\%CI: 1.044-19.441) and chest tightness (OR: 0.106; 95\%CI: 0.015-0.743) were independent factors.

\subsection{Association between co-infection and negative conversion}

All patients achieved negative conversion, with the median duration of negative conversion among all COVID19 patients was 13 days (IQR: 10-18). Moreover, it was 13 days (IQR: 10-15) in patients with bacterial co-infection, as well as 10 days (IQR: 7.5-16.5) in patients with viral co-infection. Results from multivariate Cox regression model revealed that after controlling independent factors reported in the previous study, such as age older than 45 years and chest tightness, co-infection of other respiratory pathogens also significantly associated with negative conversion of SARS-CoV-2 RNA (Hu et al., 2020). Co-infection of only bacteria, only viruses and mixed of them were all able to promote negative conversion of COVID-19, but the promotion was different in terms of different types of co-infection. As shown in Figure 2 , the strongest promotion for negative conversion was detected with co-infection of only viruses (HR: 4.039; 95\%CI: 1.238-13.177), and the weakest was found for co-infection of only bacteria (HR: 2.909; 95\%CI: 1.308-6.471). Interestingly, the promotion in co-infection of mixed bacteria and viruses was between co-infection of only bacteria and only viruses, and its $\mathrm{HR}$ was 3.242 with $95 \% \mathrm{CI}$ ranging from 1.171 to 8.977 .

\section{Discussion}

Owing to the important implication of SARS-CoV-2 co-infection for COVID-19 management, we found a large proportion of co-infection with other respiratory pathogens among COVID-19 patients in Qingdao, China. Meanwhile, we determined independent factors associated with co-infection by univariate and multivariate analysis. Besides, negative conversion of SARS-CoV-2 RNA was considered as associated variable of prognosis to evaluate the impact of co-infection on COVID-19 patients. Our findings suggested the distribution of co-infection in COVID-19, and provided the evidence that co-infection of only bacteria, only viruses and mixed of them could variously affect the COVID-19. A reported rate of COVID-19 co-infection with 39 pathogen detection was $94.2 \%$ (virus $31.5 \%$, bacteria $91.8 \%$ ) from Zhu (Zhu et al., 2020), as well as other reported rates of co-infected pathogens from 13.5\% to 20.7\% (Kim et al., 2020; Wang et al.). In our study, the co-infection rate of COVID-19 patients was $76.4 \%$ (virus $23.6 \%$, bacteria $63.6 \%$ ), which stayed similar level in comparison with other studies. In order to further verify whether the high rate of COVID-19 co-infection related to SARS-CoV-2 infection, we collected pneumonia cases in fever clinics considered as 
suspected cases of COVID-19, including 178 febrile outpatients with pneumonia who admitted to the local hospitals in Qingdao at the same time. As shown in Figure 3, the common pathogens in COVID-19 patients and pneumonia cases were almost the same, including SP, HI, MC and IFV-B, IFV-A. However, there was a significant difference for rates of co-infection between COVID-19 patients and pneumonia cases $(\mathrm{P}<0.05)$, and the rate of co-infection in COVID-19 patients was four times of the co-infection rate of pneumonia cases $(19.1 \%)$.

To our best knowledge, this has been the first study focused on independent factors associated with SARS$\mathrm{CoV}-2$ co-infection. Based on co-infection was no associated with disease severity, we further analyzed in terms of separately bacteria and viruses to determine characteristics of co-infection in COVID-19 patients. Among all co-infection patients, $83.3 \%$ cases were detected for bacterial co-infection, which were more than twice for viral co-infection (31.0\%). For co-infection of bacteria, the most common bacterial pathogens were SP and HI. Results from multivariate Logistic model revealed that over $70 \%$ of neutrophils proportion was an independently factor of co-infection of bacteria, which positively associated with bacterial co-infection (OR: 4.563; 95\%CI: 1.116-18.648). Moreover, for co-infection of viruses, the most common viral pathogen is INF-B. After multivariate Logistic regression analysis, fever and chest tightness were independently factors of co-infection of viruses. Fever (OR: 4.506; 95\% CI: 1.044-19.441) was positively associated with co-infection of viruses, whereas chest tightness (OR: 0.106; 95\%CI: 0.015-0.743) was negatively associated. These findings suggest the need to conduct comprehensive microbiologic surveys and clinical evaluation for other respiratory pathogens in COVID-19 patients, and clinicians should pay more attention on the co-infection for confirmed COVID-19 cases, which have great implications for COVID-19 treatment. Additionally, these independent factors may help clinicians to identify keys for co-infection prevention in COVID-19 patients.

At present, there has been limit study reporting the impact of co-infection on COVID-19 patients, which mostly focused on the descriptive characteristics of co-infection. However, this study has firstly provided evidence that co-infection could impact on COVID-19, which presents the association with negative conversion of SARS-CoV-2 RNA. Results suggested that co-infection was associated with a promoted shedding of SARS-CoV-2 in COVID-19 patients. Compared with COVID-19 patients without co-infection, patients with co-infection could promote the duration of negative conversion of SARS-CoV-2 RNA, and the effect of promotion varies from different types of co-infection pathogens. Results from multivariate Cox regression revealed that among all types of co-infection, The strongest promotion for negative conversion was detected with co-infection of only viruses (HR: 4.039; 95\% CI: 1.238-13.177), and the weakest was found for co-infection of only bacteria (HR: 2.909; 95\% CI: 1.308-6.471). Interestingly, the promotion in co-infection of mixed bacteria and viruses was between co-infection of only bacteria and only viruses, and its HR was 3.242 with 95\% CI ranging from 1.171 to 8.977 . However, there is no clear explanation for these findings. One of the potential explanation for this phenomenon may attribute to combination therapy. Although there has been no treatment guideline for co-infection in COVID-19, and the recommendations from different organizations are also inconsistent, combination therapy with non-anti-SARS-CoV-2 agents in co-infected COVID-19 patients has been seriously considered. In China, antibiotic therapy was recommended under different situations for COVID-19 patients in whom co-bacterial infection cannot be ruled out. Empirical antibiotic, such as amoxicillin, azithromycin, or fluoroquinolones, was recommended for mild cases, but broad-spectrum antibiotic covering all possible pathogens was suggested for severe cases (Jin et al., 2020). Based on the limited data of the present work, it remains unclear which antimicrobial agents should be empirically prescribed in patients with COVID-19. In addition, antimicrobial stewardship program should be implemented to prevent the rising rates of antimicrobial resistance could be caused by an increase in inappropriate antibiotic use for viral pneumonia (Huttner et al., 2020). Besides combination therapy, another potential explanation may attribute to much antagonistic effect of bacteria for SARS-CoV-2 than it of other viruses. Our findings suggest that there may be interaction in viral or bacterial replication and amplification in COVID-19 co-infection. As of now, there has been no evidence explain this phenomenon. Wilks et al proposed that defence system of host, as a supraorganism, contained commensal bacteria and immune system to against bacterial and viral pathogens (Wilks et al., 2012). Several researchers supported the view that the microbiota could inhibit viral replication, and affect virally induced pathogenesis (Domínguez-Díaz C et al., 2019; Khan R et al., 2019, 
Shi $\mathrm{Z}$ et al., 2018). Moreover, viruses in multiple infections can interact with each other in different ways, with different results such as antagonism (Mascia et al., 2016). These views may be useful in explaining our findings.

It is notable that there are several limitations of this study. A relatively small number of COVDI-19 cases were evaluated in comparison with other studies. There were 55 discharged patients of COVID-19 in Qingdao during the study period. Although we firstly assessed the association between co-infection and negative conversion of SARS- CoV-2 RNA, further studies regarding the impact of co-infection on COVID-19 prognosis should be warranted. In our study, we try to identify the interaction between SARS-CoV-2 and other respiratory pathogens by the duration of negative conversion. The timeline of viral load of SARS-CoV-2 and other respiratory pathogens during the disease period may be also an effective variable for better understanding the interaction with co-infected pathogens. Due to lack of continuous Ct values of all pathogens, we are unable to analyze in this aspect, but future studies should pay more attention doing this work.

\section{Conclusion}

A higher proportion of bacterial co-infection is detected in comparison with viral co-infection among COVID19 patients in Qingdao, China. Over 70\% of neutrophils proportion, fever and chest tightness are independent factors associated with specific co-infection in SARS-CoV-2 infection. Additionally, different types of coinfection could variously result in a promoted viral shedding of COVID-19.

\section{Funding}

This research did not receive any specific grant from funding agencies in the public, commercial, or not-forprofit sectors.

\section{Declaration of competing interest}

The authors declare that they have no known competing financial interests or personal relationships that could have appeared to influence the work reported in this paper.

\section{Acknowledgements}

We are deeply thankful to all health-care workers involved in the diagnosis and treatment of patients in Qingdao.

\section{Data availability statement}

The data that support the findings of this study will be made available from the corresponding author on reasonable request. It will be necessary to provide a proposal with detailed description of study objectives and statistical analysis plan for evaluation of the reasonability of requests. Additional materials might also be needed during the process of evaluation. Deidentified participant data will be provided after approval from the corresponding authors.

\section{Reference}

Alfaraj, S.H., Al-Tawfiq, J.A., Altuwaijri, T.A., Memish, Z.A. (2017). Middle East Respiratory Syndrome Coronavirus and pulmonary tuberculosis coinfection: implications for infection control. Intervirology. 60, $53-5$.

Arabi, Y.M., Al-Omari, A., Mandourah, Y., Al-Hameed, F., Sindi, A.A., Alraddadi, B., Shalhoub, S., Almotairi, A., Khatib, K., Abdulmomen, A., Qushmaq, I., Mady, A., Solaiman, O., AI-Aithan, A.M., Raddadi, A., Ragab, A., Mekhlafi, G.A.A., Harthy, A.A., Kharaba, A., Ahmadi, M.A., Sadat, M., Murairi, H.A., Qasim, E.A., Jose, J., Nasim, M., Ai-Dawood, A., Merson, L., Fowler, R., Hayden, F.G., Balkhy, H.H. (2017). Critically ill patients with the Middle East Respiratory Syndrome: a multicenter retrospective cohort study. Crit. Care. Med. 45, 1683-95.

Domínguez-Díaz, C., García-Orozco, A., Riera-Leal, A., Padilla-Arellano, J.R., Fafutis-Morris, M. (2019) Microbiota and Its Role on Viral Evasion: Is It With Us or Against Us?. Front Cell Infect Microbiol. 9, 256. 
Hu, X., Xing, Y., Jia, J., Ni, W., Liang, J., Zhao, D., Song, X., Gao, R., Jiang, F. (2020). Factors associated with negative conversion of viral RNA in patients hospitalized with COVID-19. Sci. Total. Environ. 728,138812 .

Hu, X., Xing, Y., Ni, W., Zhang, F., Lu, S., Wang, Z., Gao, R., Jiang, F. (2020). Environmental contamination by SARS-CoV-2 of an imported case during incubation period. Sci. Total. Environ. 742,140620.

Huttner, B., Catho, G., Pano-Pardo, J.R., Pulcini, C., Schouten, J. (2020). COVID-19: don't neglect antimicrobial stewardship principles! Clin. Microbiol. Infect.26, 808-810.

Jin, Y., Cai, L., Cheng, Z., Cheng, H., Deng, T., Fan, Y. (2020). For the Zhongnan hospital of Wuhan university novel coronavirus management and research Team, evidence-based medicine Chapter of China international exchange and promotive association for medical and Health care (CPAM). A rapid advice guideline for the diagnosis and treatment of 2019 novel coronavirus (2019-nCoV) infected pneumonia (standard version). Mil. Med. Res. 7, 4.

Khan, R., Petersen, F.C., Shekhar, S. (2019). Commensal Bacteria: An Emerging Player in Defense Against Respiratory Pathogens. Front. Immunol. 10, 1203.

Kim, D., Quinn, J., Pinsky, B., Shah, N.H., Brown, I. (2020). Rates of Co-infection Between SARS-CoV-2 and Other Respiratory Pathogens. JAMA. 323, 2085-2086.

Lai, C.C., Wang, C.Y., Hsueh, P.R. (2020). Co-infections among patients with COVID-19: The need for combination therapy with non-anti-SARS-CoV-2 agents? J. Microbiol. Immunol. Infect. doi: 10.1016/j.jmii.2020.05.013. [Epub ahead of print].

Lee, N., Chan, P.K., Yu, I.T., Tsoi, K.K., Lui, G., Sung, J.J., Cockram, C.S. (2007). Co-circulation of human metapneumovirus and SARS-associated coronavirus during a major nosocomial SARS outbreak in Hong Kong. J. Clin. Virol. 40, 333-7.

Mascia, T., Gallitelli, D. (2016). Synergies and antagonisms in virus interactions. Plant. Sci. 252, 176-192.

National Health Commission of the People's Republic of China (NHCPRC), (2020a). Diagnosis and treatment plan of Corona virus disease 2019 (tentative fifth edition). [Assessed 5 Feb, 2020]. Availbe from. http://www.nhc.gov.cn/yzygj/s7653p/202002/3b09b894ac9b4204a79db5b8912d4440.shtml.

National Health Commission of the People's Republic of China (NHCPRC), (2020b). Diagnosis and Treatment Plan of Corona Virus Disease 2019 (tentative sixth edition). [Assessed Feb 19, 2020]. Availbe from:. http://www.nhc.gov.cn/yzygj/s7653p/202002/8334a8326dd94d329df351d7da8aefc2.shtml.

National Health Commission of the People's Republic of China (NHCPRC), (2020c). Diagnosis and Treatment Plan of Corona Virus Disease 2019 (tentative seventh edition). [Accessed 4 March, 2020]. Availbe from. http://www.nhc.gov.cn/yzygj/s7653p/202003/46c9294a7dfe4cef80dc7f5912eb1989.shtml.

Shi, Z., Gewirtz, A.T. (2018). Together Forever: Bacterial-Viral Interactions in Infection and Immunity. Viruses. $10(3), 122$.

Wang, M., Wu, Q., Xu, W., Qiao, B., Wang, J., Zheng, H.,Jiang, S., Mei, J., Wu, Z., Deng, Y., Zhou, F., Wu, W., Zhang, Y., Lv, Z., Huang, J., Guo, X., Feng, L., Xia, Z., Li, D., Xu, Z., Liu, T., Zhang, P., Tong, Y., Li, Y. (2020). Clinical diagnosis of 8274 samples with 2019-novel coronavirus in Wuhan. medRxiv. doi.org/10.1101/2020.02.12.20022327. [Online ahead of print].

Wilks, J., Beilinson, H., Golovkina, T.V. (2013). Dual role of commensal bacteria in viral infections. Immunol Rev. 255(1), 222-229. doi:10.1111/imr.12097.

World Health Orgnization (WHO), (2020). Novel Coronavirus (COVID-19) situation reports. 2020. [Assessed 3 June, 2020]. Available from: https://www.who.int/emergencies/diseases/novel-coronavirus-2019/situationreports. 
Zahariadis, G., Gooley, T.A., Ryall, P., Hutchinson, C., Latchford, M.I., Fearon, M.A., Jamieson, F.B., Richardson, S., Kuschak, T., Mederski, B. (2006). Risk of ruling out severe acute respiratory syndrome by ruling in another diagnosis: variable incidence of atypical bacteria coinfection based on diagnostic assays. Canc. Res. J. 13, 17-22.

Zhu, X., Ge, Y., Wu, T., Zhao, K., Chen, Y., Wu, B., Zhu, F., Zhu, B., Cui, L. (2020). Co-infection with respiratory pathogens among COVID-2019 cases. Virus. Res. 285, 198005.

\section{Figure legends}

Figure 1 The rates of co-infected pathogens in SARS-CoV-2 positive samples (A: single virus classification; B: combined viruses classification)

Figure 2 Results of association between co-infection and negative conversion from Cox regression

Figure 3 The comparison of co-infection between COVID-19 and pneumonia cases (A: overall rate of coinfection; B: rate of infection for common respiratory pathogens)

\section{Hosted file}

Table.docx available at https://authorea.com/users/354370/articles/477963-co-infectionof-other-respiratory-pathogens-in-covid-19-associated-factors-analysis-and-prognosticimpact-assessment 


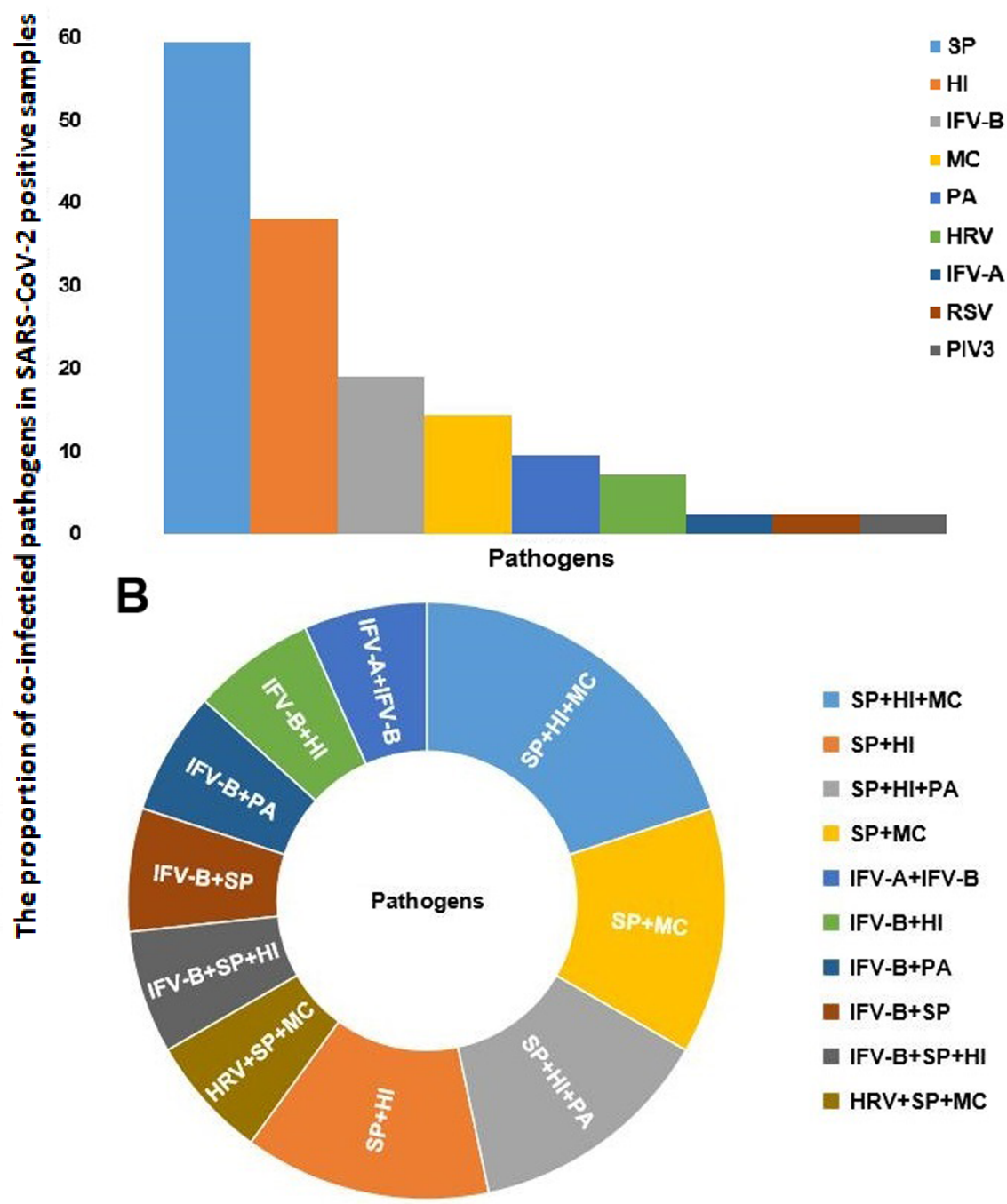

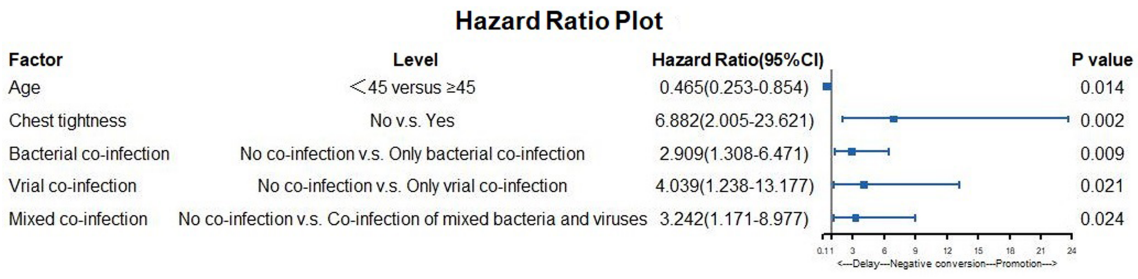



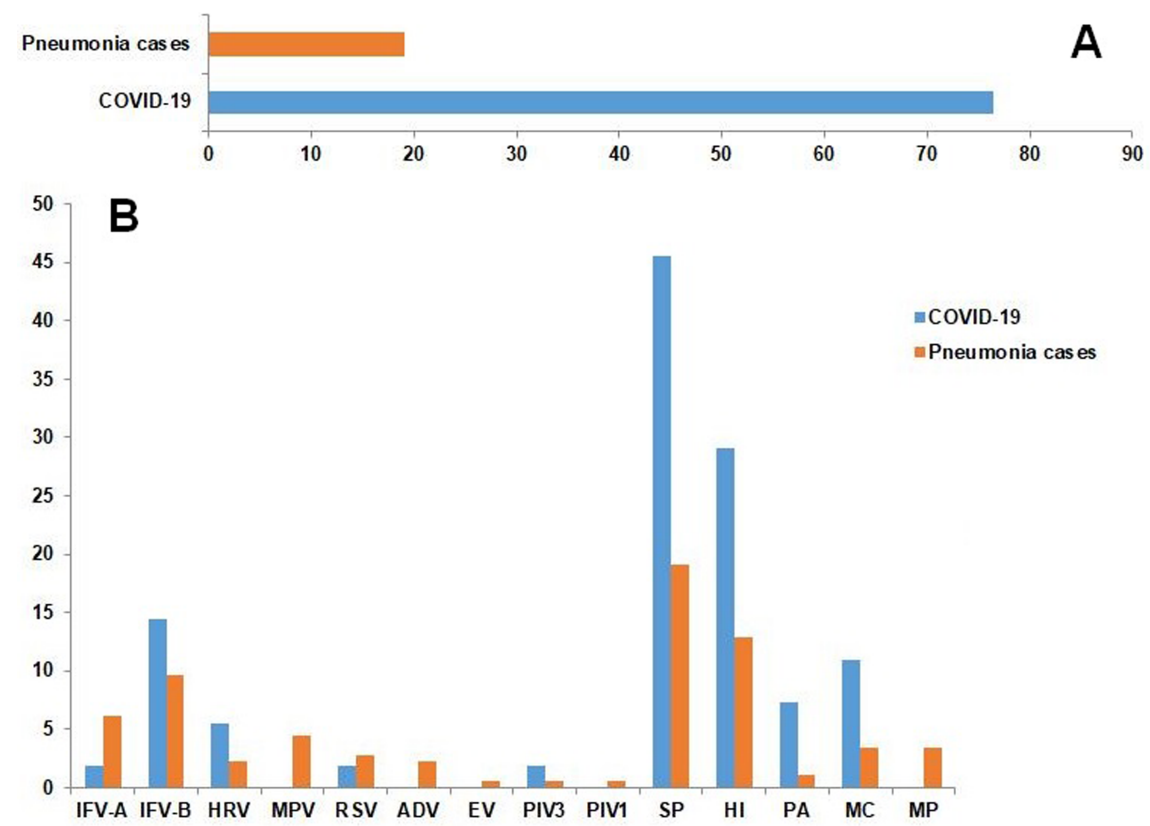